\title{
Hadron spectroscopy and interactions from lattice QCD
}

\author{
Sasa Prelovsek ${ }^{1,2, \star}$ \\ ${ }^{1}$ Department of Physics, University of Ljubljana, Jadranska 19, Ljubljana, Slovenia \\ ${ }^{2}$ Jozef Stefan Institute, Jamova 39, 1000 Ljubljana, Slovenia
}

\begin{abstract}
Lattice QCD approach to study the hadronic resonances and exotic hadrons is described at an introductory level. The main challenge is that these states decay strongly via one or more decay channels, and they often lie near thersholds. Specific results for conventional and exotic hadrons are shown to illustrate the current status.
\end{abstract}

\section{Introduction}

Lattice QCD provides very accurate and reliable results for hadrons that lie well below strong-decay threshold and do not decay strongly. Over past few years, the lattice community provided also rigorous results for the resonances that can decay strongly via one-decay channel $R \rightarrow H_{1} H_{2}$. Interesting shallow bound states that lie just below $\mathrm{H}_{1} \mathrm{H}_{2}$ threshold were also investigated. Both require the simulation of $H_{1} H_{2}$ scattering and extraction of the underlying scattering matrix. A resonance that can strongly decay to several channels presents an even more challenging problem, which implies for the majority of the exotic hadrons. First lattice results were obtained very recently on this front as well. Here I illustrate the lattice approach to this problem in the introductory manner. Only few selected results are shown to illustrate various types of cases, while this is not a review of available results.

\section{Lattice methodology}

The physics information on a hadron (below, near or above threshold) is commonly extracted from the discrete energy spectrum in lattice QCD. The physical system for given quantum numbers is created from the vacuum $|\Omega\rangle$ using interpolator $O_{j}^{\dagger}$ at time $t=0$ and the system propagates for time $t$ before being annihilated by $O_{i}$. To study a meson or an exotic-meson with given $J^{P}$, one can use $O \simeq \bar{q} \Gamma q$, two-meson interpolators $O=\left(\bar{q} \Gamma_{1} q\right)\left(\bar{q} \Gamma_{2} q\right),\left(\bar{q} \Gamma_{1} q\right)\left(\bar{q} \Gamma_{2} q\right)$ and $O=\left[\bar{q} \Gamma_{1} \bar{q}\right]\left[q \Gamma_{2} q\right]$ with desired quantum numbers. After the spectral decomposition the correlators are expressed in terms of the energies $E_{n}$ of eigenstates $|n\rangle$ and their overlaps $Z_{j}^{n}$

$$
C_{i j}(t)=\left\langle\Omega\left|O_{i}(t) O_{j}^{\dagger}(0)\right| \Omega\right\rangle=\sum_{n} Z_{i}^{n} Z_{j}^{n *} e^{-E_{n} t}, \quad Z_{i}^{n} \equiv\left\langle\Omega\left|O_{i}\right| n\right\rangle .
$$

The correlators are evaluated on the lattice and their time-dependence allows to extract $E_{n}$ and $Z_{n}^{i}$ $[1,2]$.

\footnotetext{
^e-mail: sasa.prelovsek@ijs.si
} 
The energy eigenstates $|n\rangle$ are predominantly "one-meson" states (e.g. $\left.\chi_{c 1}(1 P)\right)$ or predominantly "two-meson" states (e.g. $\left.D \bar{D}^{*}\right)$ - in interacting theory they are mixtures of those. Two-meson states have a discrete spectrum due to the periodic boundary condition on the finite lattices. If they do not interact, then the momenta of each meson is $\vec{p}=\frac{2 \pi}{L} \vec{N}$ with $\vec{N} \in N^{3}$, and the non-interacting energies of $M_{1}(\vec{p}) M_{2}(-\vec{p})$ are $E^{n . i .}=E_{1}(p)+E_{2}(p)$ with $E_{1,2}(p)=\left(m_{1,2}^{2}+p^{2}\right)^{1 / 2}$. The energies $E_{n}$ extracted from the lattice are slightly shifted in presence of the interaction and the shift provides rigorous information on the scattering matrix, as discussed in Section 4. In experiment, two-meson states correspond to the two-meson decay products with a continuous energy spectrum.

\section{Hadrons well below strong-decay threshold}

Masses are straightforwardly determined for the hadrons that can not decay strongly and lie well below strong decay threshold. These masses $m_{i}=E_{i}(P=0)$ are extracted from the energies obtained with $\bar{q} q$ or $q q q$ interpolating fields, which are extrapolated $a \rightarrow 0, V \rightarrow \infty$ and $m_{q} \rightarrow m_{q}^{\text {phys }}$. The resulting masses for all such hadrons (i.e. $\pi, K, D, B, B^{*}, B_{s}, J / \psi, \eta_{b}, n, p, \Lambda, \ldots$ ) are in very good agreement with the experimental measurements. The main remaining uncertainty is the omission of charm and beauty annihilation for heavy quarkonia.

\section{Scattering approach to resonances and bound states}

Most of the hadrons are resonances, they are located above strong decay threshold(s) and decay strongly. In the energy region near or above threshold, the masses of bound-states and resonances have to be inferred from the infinite-volume scattering matrix of the one-channel (elastic) or multiplechannel (inelastic) scattering. The simplest example is a one-channel elastic scattering in partial wave $l$, where the scattering matrix is parametrized in terms of the phase shift $\delta_{l}(p)$ and satisfies unitarity $S S^{\dagger}=1$

$$
S(E)=e^{2 i \delta_{l}(E)}, \quad S(E)=1+2 i T(E), \quad T(E)=\frac{1}{\cot \left(\delta_{l}(E)\right)-i} .
$$

Lüscher has shown that the energy $E$ of two-meson eigenstate in finite volume $L$ gives the elastic phase shift $\delta(E)$ at that energy in infinite volume [3]. This relation and its generalizations are at the core of extracting rigorous information about the scattering from the recent lattice simulation. It leads $\delta(E)$ only for specific values of $E$ since since spectrum of two-meson eigenstates is discrete. The $\delta(E)$ or $T(E)$ (2) provide the masses of resonances and bound states:

- In the vicinity of a hadronic resonance with mass $m_{R}$ and width $\Gamma$, the cross section $\sigma \propto|T(p)|^{2}$ has a Breit-Wigner-type shape with $\delta\left(s=m_{R}^{2}\right)=\frac{\pi}{2}$

$$
T(p)=\frac{-\sqrt{s} \Gamma(p)}{s-m_{R}^{2}+i \sqrt{s} \Gamma(p)}=\frac{1}{\cot \delta(p)-i}, \quad \Gamma(p)=g^{2} \frac{p^{2 l+1}}{s} .
$$

The fit of $\delta_{l}(p)$ renders $m_{R}$ and $g$ or $\Gamma$. It is customary to compare $g$ rather than $\Gamma$ to experiment, since $\Gamma$ depends on the phase space. Lüsher's approach has been verified on several conventional mesonic resonances.

- The bound state $(B)$ in $M_{1} M_{2}$ scattering is realized when $T(p)$ has a pole at $p_{B}^{2}<0$ or $p_{B}=i\left|p_{B}\right|$

$$
T=\frac{1}{\cot \left(\delta_{l}\left(p_{B}\right)\right)-i}=\infty, \quad \cot \left(\delta\left(p_{B}\right)\right)=i, \quad m_{B}=E_{H_{1}}\left(p_{B}\right)+E_{H_{2}}\left(p_{B}\right) .
$$

The location of an s-wave shallow bound state can be obtained by parametrizing $\delta_{0}$ near threshold and finding $p_{B}$ which satisfies $\cot \left(\delta\left(p_{B}\right)\right)=i$. 

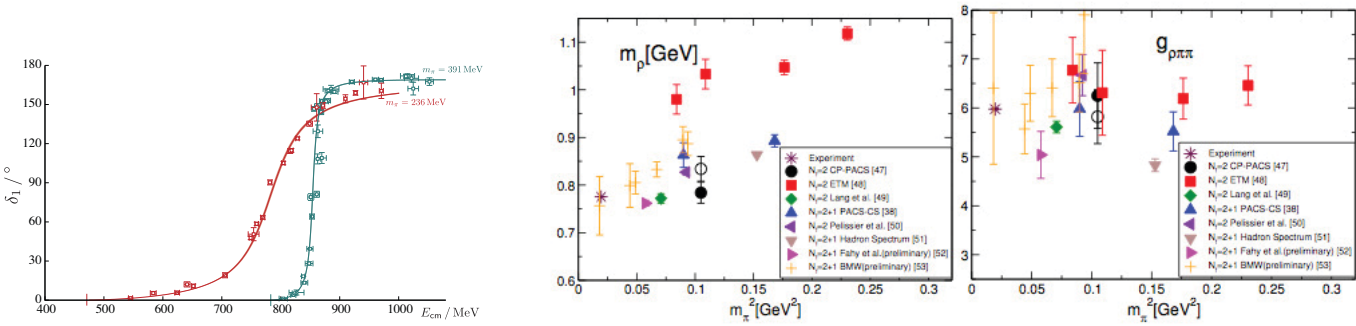

Figure 1. Left: The $\pi \pi$ phase shift in the $\rho$-meson channel from the simulation by the Hadron Spectrum Collaboration $[4,5]$. Right: The compilation [6] of the masses and widths for the $\rho$ meson from recent lattice simulations, where the $g$ parametrizes the width as $\Gamma=g^{2} p^{3} /(6 \pi s)$.
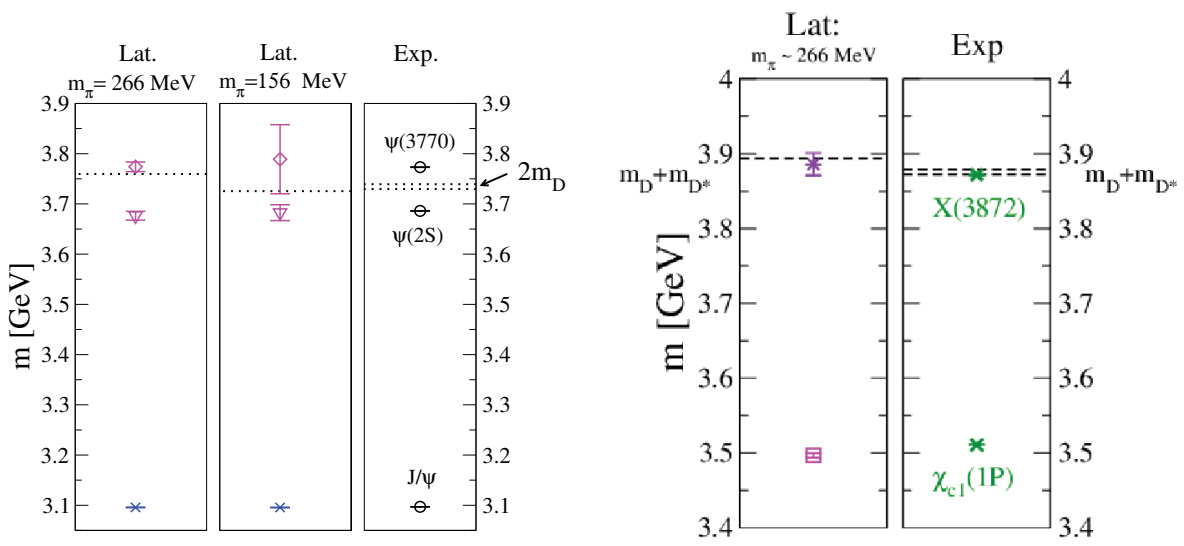

Figure 2. Left: The lattice spectrum of the vector charmonia from [7]: the diamond denotes the resonance mass of $\psi(3770)$, while the triangle denotes the pole mass of the bound state $\psi(2 S)$; both are obtained from $D \bar{D}$ scattering matrix. Right: The location of $X(3872)$ with $I=0$ which emerges as shallow bound state in a simulation of $D \bar{D}^{*}$ scattering that includes also diquark antidiqark interpolating fields [8].

\section{Selected resonances from one-channel scattering}

$\rho$ meson: Only one resonance - namely the $\rho$ meson - has been rigorously studied by a number of lattice collaborations, reviwed in [6]. Figure 1 shows an example of extracted $\pi \pi$ phase shift for $L=1$ and $I=1$, obtained by the Hadron Spectrum Collaboration $[4,5]$ for two pion masses. The Breit-Wigner-type fit renders $\rho$ resonance mass and width [4] shown by the brown triangle in Fig. 1, where other lattice results are also shown.

Charmonium resonances: The charmonium spectrum above open charm threshold contains a number of exotic candidates. It is important to verify the approach on the conventional states before addressing the exotic states. Until recently, all charmonia above open-charm threshold were treated ignoring the strong decay to a pair of charmed mesons. The first exploratory simulation aimed at determining the masses as well as the decay widths of these resonances was presented in [7]. The Breit-Wigner-type fit of the $D \bar{D}$ phase shift in $p$-wave leads to the resonance $\psi(3770)$ mass in agreement with experiment, as shown in Fig. 5. The extracted $\psi(3770) \rightarrow D \bar{D}$ decay width $\Gamma=g^{2} p / s$ 

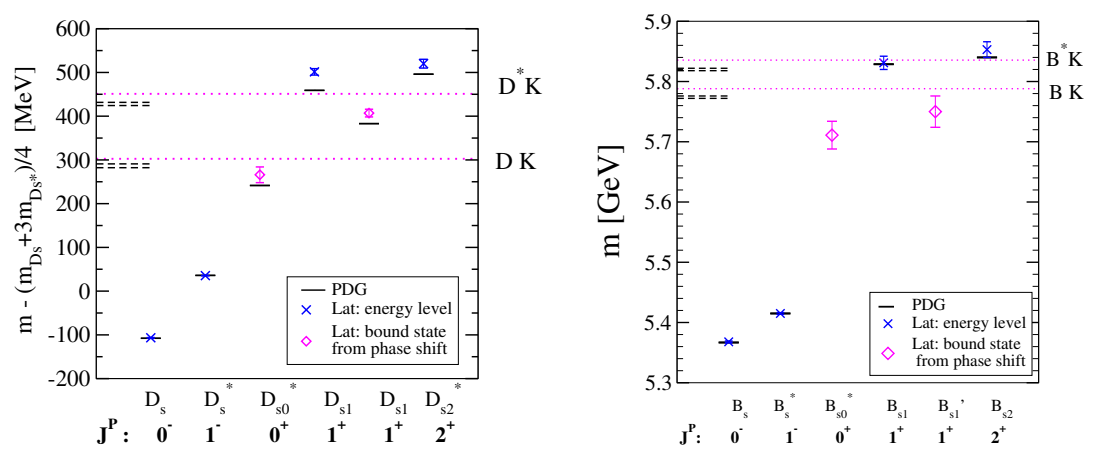

Figure 3. The spectra of $D_{s}[9,10]$ and $B_{s}[11]$ mesons resulting from $N_{f}=2+1$ lattice simulation on PACS-CS ensembles with $m_{\pi} \simeq 156 \mathrm{MeV}$. The black horizontal lines are experimental masses.

with $g_{m_{\pi}=266 \mathrm{MeV}}^{l a t}=19.7 \pm 1.4$ is also close to $g^{\exp }=18.7 \pm 1.4$. The $\psi(2 S)$ in Fig. 5 appears as a bound state pole below threshold. In the scalar channel, only the ground state $\chi_{c 0}(1 P)$ is understood and there is no commonly accepted candidate for its first excitation $\chi_{c 0}(2 P)$. The lattice results on the $D \bar{D}$ scattering in s-wave [7] leave open puzzles and call for further work on this problem.

\section{Selected shallow bound states from one-channel scattering}

Scalar and axial mesons $D_{s}$ and $B_{s}$ : The quark models expected $D_{s 0}^{*}(2317)$ and $D_{s 1}(2460)$ above $\overline{D K}$ and $D^{*} K$ thresholds, but they were experimentally found slightly below them. These are one of few shallow bound states in the meson sector, and therefore deserve special attention. The first lattice simulation that takes the effect of these thresholds into account was presented in $[9,10]$. It employs $D K$ and $D^{*} K$ interpolating fields in addition to the $\bar{s} c$. The $D^{(*)} K$ phase shift is extracted and then interpolated in the region close to threshold. A pole of the scattering matrix is found below the thresholds where the relation (4) is satisfied. The poles are related to $D_{s 0}^{*}(2317)$ and $D_{s 1}(2460)$ bound states and are close to the masses of experimentally observed states, as shown in Fig. 3 . The scalar and axial $B_{s}$ mesons have not been discovered in experiment yet, and in this case we made a prediction for their masses using analogous simulation of $B^{(*)} K$ scattering. We find poles related to $B_{s 0}$ and $B_{s 1}$ mesons below $B K$ and $B^{*} K$ thresholds [11], as shown in Fig. 3.

$X(3872)$ : The $X(3872)$ lies experimentally on $D^{0} \bar{D}^{0 *}$ threshold and its existence on the lattice can not be established without taking into account the effect of this threshold. This was first done by simulating $D \bar{D}^{*}$ scattering in [12], where a pole in $D \bar{D}^{*}$ scattering matrix was found just below the threshold in $I\left(J^{P C}\right)=0\left(1^{++}\right)$channel (4). The pole was associated with $X(3872)$ [12] and was also confirmed by a simulation with HISQ action [13]. The more recent simulation [8] included also the diquark anti-diquark interpolating fields and the location of the $X(3872)$ pole is shown in Fig. 5.

The lattice study [8] investigated which Fock components are essential for appearance of $X(3872)$ with $I=0$ on the lattice. The energy eigenstate related to $X(3872)$ appears in the simulation only if $D \bar{D}^{*}$ as well as $\bar{c} c$ interpolating fields are employed. The $X(3872)$ does not appear in absence of $\bar{c} c$ interpolators, even if (localized) interpolators $[\bar{c} \bar{q}]_{3_{c}}[c q]_{\overline{3}_{c}}$ or $[\bar{c} \bar{q}]_{6_{c}}[c q]_{\bar{b}_{c}}$ are in the interpolator basis. This indicates that $\bar{c} c$ Fock component is most likely more essential for $X(3872)$ than the diquark-antidiquark one. 
Pentaquark bound state: The NPLQCD collaboration finds an interesting evidence for a $\eta_{c} N$ bound state approximately $20 \mathrm{MeV}$ below $\eta_{c} N$ threshold [14] ( $N$ denotes nucleon). To my knowledge, this is the only pentaquark candidate containing $\bar{c} c$ from lattice studies up to now. As the simulation is done at $S U(3)$ flavour symmetric point corresponding to $m_{\pi} \simeq 800 \mathrm{MeV}$, it is not clear yet whether this bound state persists to physical $m_{\pi}$. LHCb has recently found two pentaquark resonances in $J / \psi p$, located about $400 \mathrm{MeV}$ above threshold [15]. The lattice simulation of those is much more challenging due to several open channels and has not been performed yet.

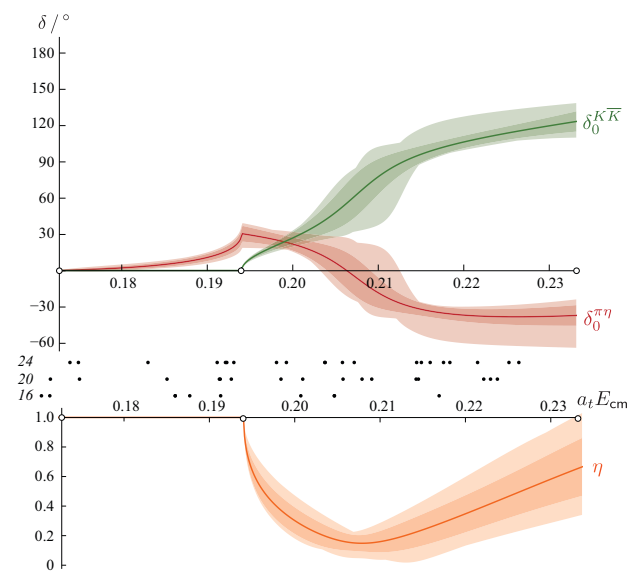

Figure 4. The phases and the inelasticity of the $K \bar{K}-\pi \eta$ coupled channel scattering in the channel where $a_{0}$ resonance appears in experiment. The results are obtained from a $N_{f}=2+1$ lattice simulation at $m_{\pi} \simeq 400 \mathrm{MeV}$ by the Hadron Spectrum Collaboration [16].

\section{Towards the coupled-channel scattering and exotic hadrons}

Most of the exotic candidates are experimentally found above several thresholds, and can decay in more than one hadronic final state. This problem requires the determination of the scattering matrix for the coupled-channel scattering. This is more challenging for a rigorous treatment than a simulation of one-channel (elastic) scattering.

$a_{0}$ resonance: The coupled-channel scattering matrix was extracted from the lattice simulation for the first time only recently [18], where the generalized Lüscher's method was used. Figure 4 shows the results for the scattering phases and inelasticity that parametrize the $2 \times 2$ scattering matrix in the $a_{0}$ channel [16].

$Z_{c}^{+}(3900)$ : The lattice search for the manifestly exotic states $Z_{c}^{+}$with flavour content $\bar{c} c \bar{d} u$ and $I^{G}\left(\overline{J^{P C}}\right)=1^{+}\left(1^{+-}\right)$is very challenging since the experimental candidate can strongly decay to several final states $D \bar{D}^{*}, J / \psi \pi$ and $\eta_{c} \rho$. The corresponding $3 \times 3$ scattering matrix has never been determined using the reliable Lüscher's method.

The HALQCD collaboration used the HALQCD approach to extracted the coupled-channel scattering matrix, which is not commonly accepted as an exact treatment, and has not been verified on the conventional resonances. First the potential $V_{\pi J / \psi-\pi J / \psi}(r)$ related to Nambu-Bethe-Salpeter equation 

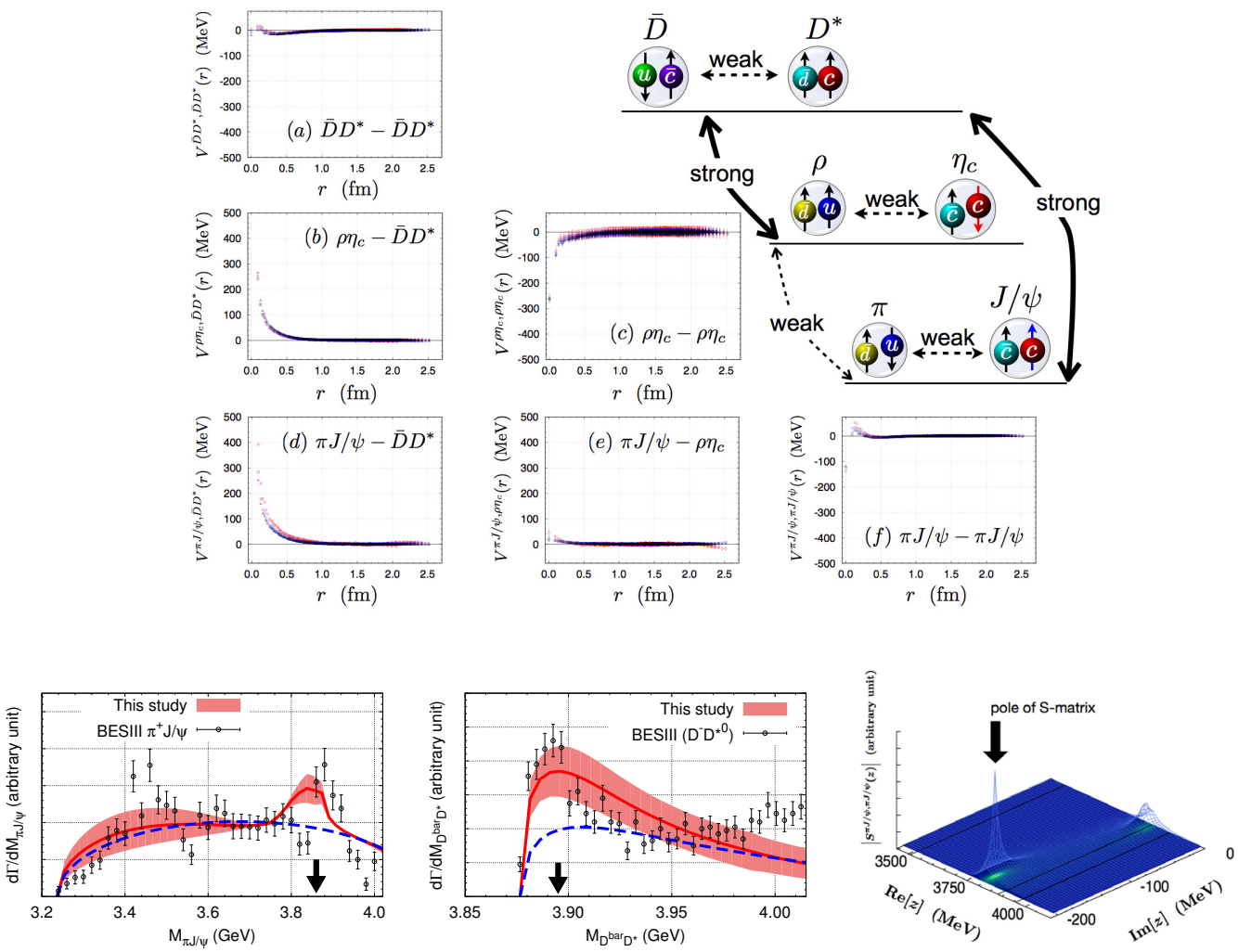

Figure 5. The results related to $Z_{c}^{+}(3900)$ from a lattice simulation [17], based on the HALQCD method. Top: potentials $V(r)$ for three channels. Bottom left: red lines present the lattice QCD results for $Y(4260) \rightarrow J / \psi \pi \pi$ and $Y(4260) \rightarrow D \bar{D}^{*} \pi$ invariant masses, while blue lines present results if $J / \psi \pi-D \bar{D}^{*}$ coupling is turned off. Bottom right: Poles of the scattering matrix in the complex energy plane.

is determined between the $J / \psi$ and $\pi$ as a function of their separation $r$ [17]. This is presented in Fig. 5 together with potential for the other two channels, as well as potential corresponding to coupling between different channels according to coupled-channel HALQCD formalism [19]. The off-diagonal potential between channels $\pi J / \psi$ and $D \bar{D}^{*}$ is larger than other potentials, which seems to indicate a sizable coupled channel effect near $D \bar{D}^{*}$ threshold. The potentials render $3 \times 3$ scattering matrix for three coupled channels [17]. This is then used to determine the three body decay $Y(4260) \rightarrow J / \psi \pi \pi$ and $Y(4260) \rightarrow D \bar{D}^{*} \pi$ in a semi-phenomenological way. The result in Fig. 5 indeed shows a peak around $Z_{c}$ mass. If the coupling between $J / \psi \pi$ and $D \bar{D}^{*}$ is turned off, the peak disappears as shown by blue dashed line. It indicates that the coupling of $J / \psi \pi$ and $D \bar{D}^{*}$ channels seems to be crucial for the existence of $Z_{c}$. This needs to be verified also by the Lüscher's method.

The first step towards the treatment of this problem with the Lüscher method is to determine the energies of eigenstates for the $Z_{c}$ system. This has been done in $[13,20]$. The number of lattice eigenstates in the relevant energy region was found to be equal to the number of expected two-hadron states in the absence of interactions, and no additional eigenstate was found. This suggests that $Z_{c}$ is perhaps not a resonance in the usual sense, i.e. it is not associated with a pole above $D \bar{D}^{*}$ threshold 
near the real axis. Indeed, the HALQCD collaboration has not found such a pole, as shown in Fig. 5. More analytical work and lattice simulations are required to conclude on this problem.

$X(4140)$ : The experimental candidate $X(4140)$ with hidden strangeness was confirmed in $J / \psi \phi$ invariant mass by $\mathrm{LHCb}$ recently $[21,22]$. The amplitude analysis favors quantum numbers $J^{P C}=1^{++}$ for it. The rigorous treatment would require extraction of the scattering matrix for at leat two channels $J / \psi \phi$ and $D_{s} \bar{D}_{s}^{*}$. The simplified lattice search was performed in this channel with $D_{s} \bar{D}_{s}^{*}, J / \psi \phi,[\bar{c} \bar{s}][c s]$ interpolators. The number of lattice eigenstates in the relevant energy region was found to be equal to the number of expected two-hadron states in the absence of interactions. No additional eigenstate was found near $4.1 \mathrm{GeV}$, which suggests that $X(4140)$ is perhaps not a resonance with a pole above $J / \psi \phi$ threshold near the real axis. The experimental study [22] found that the amplitudes could be fitted within the cusp scenario [23], which might be consistent with absence of a resonance pole near $E_{c m} \simeq m_{X}-i \Gamma_{X} / 2$.

\section{Summary}

During the past few years, the lattice QCD provided rigorous results on the hadrons that lie close and above strong-decay thresholds. Verification of the approach on the conventional hadrons is the first important step towards understanding exotic ones. Most of the experimental exotic candidates can strongly decay via several decay channels and lie near thresholds. This presents a challenge for rigorous treatment from first principles, but important encouraging steps in this direction have been accomplished.

\section{Acknowledgments}

I would like to thank C.B. Lang, D. Mohler, L. Leskovec, M. Padmanath and R. Woloshyn for pleasant collaboration on these physics problems. Support from Slovenian Research Agency ARRS under project N1-0020 is acknowledged.

\section{References}

[1] C. Michael, Nucl. Phys. B 259, 58 (1985).

[2] B. Blossier, M. DellaMorte, G. von Hippel, T. Mendes, R. Sommer, JHEP 0904, 094 (2009), 0902.1265.

[3] M. Lüscher, Nucl. Phys. B 364, 237 (1991).

[4] J.J. Dudek, R.G. Edwards, C.E. Thomas, Phys.Rev. D87, 034505 (2013), 1212 . 0830.

[5] D.J. Wilson, R.A. Briceno, J.J. Dudek, R.G. Edwards, C.E. Thomas, Phys. Rev. D92, 094502 (2015), 1507.02599.

[6] T. Yamazaki, PoS LATTICE2014, 009 (2015), 1503.08671.

[7] C.B. Lang, L. Leskovec, D. Mohler, S. Prelovsek (2015), 1503.05363.

[8] M. Padmanath, C.B. Lang, S. Prelovsek, Phys. Rev. D92, 034501 (2015), 1503.03257.

[9] D. Mohler, C. Lang, L. Leskovec, S. Prelovsek, R. Woloshyn, Phys.Rev.Lett. 111, 222001 (2013), 1308.3175.

[10] C. Lang, L. Leskovec, D. Mohler, S. Prelovsek, R. Woloshyn, Phys.Rev. D90, 034510 (2014), 1403.8103.

[11] C.B. Lang, D. Mohler, S. Prelovsek, R.M. Woloshyn, Phys. Lett. B750, 17 (2015), 1501 . 01646.

[12] S. Prelovsek, L. Leskovec, Phys.Rev.Lett. 111, 192001 (2013), 1307. 5172. 
[13] S.h. Lee, C. DeTar, H. Na, D. Mohler (Fermilab Lattice, MILC) (2014), 1411 . 1389.

[14] S.R. Beane, E. Chang, S.D. Cohen, W. Detmold, H.W. Lin, K. Orginos, A. Parreño, M.J. Savage, Phys. Rev. D91, 114503 (2015), 1410.7069.

[15] R. Aaij et al. (LHCb), Phys. Rev. Lett. 115, 072001 (2015), 1507.03414.

[16] J.J. Dudek, R.G. Edwards, D.J. Wilson (Hadron Spectrum), Phys. Rev. D93, 094506 (2016), 1602.05122.

[17] Y. Ikeda, S. Aoki, T. Doi, S. Gongyo, T. Hatsuda, T. Inoue, T. Iritani, N. Ishii, K. Murano, K. Sasaki (2016), 1602.03465.

[18] J.J. Dudek, R.G. Edwards, C.E. Thomas, D.J. Wilson (Hadron Spectrum), Phys. Rev. Lett. 113, 182001 (2014), 1406.4158.

[19] S. Aoki, B. Charron, T. Doi, T. Hatsuda, T. Inoue et al., Phys.Rev. D87, 034512 (2013), 1212.4896.

[20] S. Prelovsek, C.B. Lang, L. Leskovec, D. Mohler, Phys. Rev. D91, 014504 (2015), 1405 . 7623.

[21] R. Aaij et al. (LHCb) (2016), 1606.07895.

[22] R. Aaij et al. (LHCb) (2016), 1606.07898.

[23] E.S. Swanson, Int. J. Mod. Phys. E25, 1642010 (2016), 1504.07952. 